\title{
The effective slip length and vortex formation in laminar flow over a rough surface
}

\author{
Anoosheh Niavarani and Nikolai V. Priezjev \\ Department of Mechanical Engineering, \\ Michigan State University, East Lansing, Michigan 48824
}

(Dated: October 23, 2018)

\begin{abstract}
The flow of viscous incompressible fluid over a periodically corrugated surface is investigated numerically by solving the Navier-Stokes equation with the local slip and no-slip boundary conditions. We consider the effective slip length which is defined with respect to the level of the mean height of the surface roughness. With increasing corrugation amplitude the effective no-slip boundary plane is shifted towards the bulk of the fluid, which implies a negative effective slip length. The analysis of the wall shear stress indicates that a flow circulation is developed in the grooves of the rough surface provided that the local boundary condition is no-slip. By applying a local slip boundary condition, the center of the vortex is displaced towards the bottom the grooves and the effective slip length increases. When the intrinsic slip length is larger than the corrugation amplitude, the flow streamlines near the surface are deformed to follow the boundary curvature, the vortex vanishes, and the effective slip length saturates to a constant value. Inertial effects promote vortex flow formation in the grooves and reduce the effective slip length.

PACS numbers: 68.08.-p, 83.50.Rp, 47.61.-k, 02.70.Dh, 47.15.Rq, 47.32.Ff
\end{abstract}




\section{INTRODUCTION}

An accurate flow prediction in micro-channels is important for the optimal design and fabrication of microfluidic devices whose applications range from medicine to biotechnology [1, 2]. The boundary conditions and the surface topology are major factors affecting the flow pattern near the solid boundary and the formation of recirculation zones. The flow separation at rough surfaces can modify the wall shear stress distribution or initiate instability towards turbulence. In microfluidic channels, the vortex flow enhances the mixing efficiency [3, 4] and promotes convective heat transfer [5, 6, 7]. In cardiovascular systems, the separation region at the entrance of branching vessels may trap lipid particles which could lead to arterial diseases [8, 9, 10]. In the present study we examine the role of slip boundary condition in determining the flow properties near rough surfaces including the separation phenomena, and distribution of pressure and shear stress along the surface.

Although the validity of the no-slip boundary condition is well accepted at the macroscopic level, recent experiments [11, 12, 13, 14, 15, 16] and molecular dynamics (MD) simulations [17, 18, 19, 20, 21, 22] reported the existence of a boundary slip in microflows. The model first proposed by Navier relates the slip velocity to the rate of shear via the proportionality coefficient, the so-called slip length. The MD simulations are particularly suitable for examining the influence of molecular parameters on the microscopic slip length at the liquid/solid interface. The advantage of the MD simulations is that a detailed flow analysis can be performed at the molecular level while the explicit specification of the boundary conditions is not required. In contrast to description of the flow near boundary by means of microscopic slip length, it is convenient to characterize the flow over macroscopically rough surfaces by the effective slip length, which is defined as a distance from the level of the mean height of the surface roughness to the point where linearly extrapolated bulk velocity profile vanishes. Recent MD studies have demonstrated that the effective slip length in a flow of simple fluids [23] and polymer melts [24] over a wavy surface agrees well with hydrodynamic predictions [25, 26] when the corrugation wavelength is larger than approximately thirty molecular diameters.

The influence of surface roughness on fluid flow with either local no-slip or zero shear stress (i.e. perfect slip) boundary conditions has been extensively studied in the past decades [27, 28, 29, 30, 31, 32, 33], see also a review section in [23]. Analytical calculations have shown 
that in a shear flow over a corrugated surface with microscopic no-slip or zero shear stress conditions, the effective boundary slip is insignificant macroscopically [27, 29]. The effective no-slip boundary plane is located at the intermediate position between crests and valleys of the rough surface when the no-slip condition is imposed along the solid boundary [16, 28, 31, 34, 35]. For an arbitrary surface roughness with small amplitudes, the slip coefficient in the Navier model is proportional to the average amplitude of the wall roughness and depends on the position of the origin of the coordinate system [30]. Applying the no-slip boundary condition along the wavy surface, Tuck and Kouzoubov [31] have demonstrated that the effective slip length is inversely proportional to the corrugation wavelength and quadratically proportional to the amplitude of the surface roughness. However, the series expansion method used in [31] fails at large wavenumbers, $k a \gtrsim 0.5$, when a backflow appears inside the grooves of the substrate. The effective slip length for a flow above the surface with deep corrugations only weakly depends on the depth of the grooves [24, 28, 33]. Despite considerable analytical efforts, the relation between the vortex flow structure in deep grooves and the effective slip length has not yet been systematically investigated.

The laminar flow separation at the corrugated surface with the local no-slip boundary conditions depends on the depth of the grooves and the Reynolds number [36, 37, 38, 39, 40, 41, 42, 43]. In a creeping flow over a sinusoidal surface, the flow circulation appears in sufficiently deep grooves and, as the corrugation amplitude increases, the vortex grows and remains symmetric [41, 44, 45]. With increasing Reynolds number, the vortex flow forms even in shallow grooves, the circulation region expands, and the center of vorticity is displaced upstream [36, 39, 40, 42]. In the limit of small-scale surface roughness and for no-slip boundary conditions, the apparent slip velocity at the mean surface becomes more negative as the Reynolds number increases [31]. A noticeable change in the effective slip length was also observed at $R e \gtrsim 100$ for laminar flow over deep grooves when the local slip length is comparable to the corrugation amplitude [24]. However, the influence of the local slip condition at the curved boundary on the vortex flow formation has not been considered at finite Reynolds numbers.

This paper is focused on investigation of the effects of local slip boundary conditions and the Reynolds number on the flow structure near periodically corrugated surfaces and the effective slip length. We will show that for the Stokes flow with the local no-slip boundary condition, the effective slip length decreases with increasing corrugation amplitude and 
the flow circulation develops in sufficiently deep grooves. In the presence of the local slip boundary condition along the rough surface, the effective slip length increases and the size of the vortex is reduced but its structure remains symmetrical. The analysis of the numerical solution of the Navier-Stokes equation with the local slip condition shows that the inertial effects promote the asymmetric vortex flow formation and reduce the effective slip length.

This paper is organized as follows. The details of a continuum model and the implementation of the local slip boundary conditions are described in the next section. The analytical results for the Stokes flow over a wavy surface by Panzer et al. [26] are summarized in Sec.IIIA. The analysis of the effective slip length and the flow structure is presented in Sec.IIIB for the no-slip case and in Sec.IIIC for the finite microscopic slip. The effect of Reynolds number on the effective slip flow over a periodically corrugated surface is studied in Sec.IIID, A brief summary is given in the last section.

\section{DETAILS OF NUMERICAL SIMULATIONS}

The two-dimensional incompressible and steady Navier-Stokes (NS) equation is solved using the finite element method. The computational setup consists of a viscous fluid confined between an upper flat wall and a lower sinusoidal wall (see Fig.1). The corrugation wavelength of the lower wall is set to $\lambda$ and is equal to the system size in the $\hat{x}$ direction. The upper wall is located at $h=\lambda$ above the reference line at $a=0$, which is defined as the mean height of the surface roughness. The continuity and NS equations are

$$
\begin{gathered}
\nabla \cdot \mathbf{u}=0 \\
\rho(\mathbf{u} \cdot \nabla \mathbf{u})=-\nabla p+\mu \nabla^{2} \mathbf{u},
\end{gathered}
$$

where $\mathbf{u}=u \hat{i}+v \hat{j}$ is the velocity vector in the cartesian coordinate system, $p$ is the pressure field, $\rho$ is the fluid density, and $\mu$ is the Newtonian viscosity.

The penalty formulation is employed to avoid decoupling between the pressure and velocity fields [46]. In this method, the continuity equation is replaced with a perturbed equation

$$
\nabla \cdot \mathbf{u}=-\frac{p}{\Lambda}
$$

where $\Lambda$ is the penalty parameter, which ensures the incompressibility condition. Thus, the 
modified momentum equations in the $\hat{x}$ and $\hat{z}$ directions are

$$
\begin{aligned}
& \rho(\mathbf{u} \cdot \nabla u)=\Lambda \nabla(\nabla \cdot \mathbf{u})+\mu \nabla^{2} u \\
& \rho(\mathbf{u} \cdot \nabla v)=\Lambda \nabla(\nabla \cdot \mathbf{u})+\mu \nabla^{2} v .
\end{aligned}
$$

The advantage of the penalty formulation is the elimination of pressure and the continuity equation. The penalty parameter $\Lambda$ must be large enough so that compressibility errors are minimal. The upper bound of $\Lambda$ is determined from the condition that the viscous effects are not destroyed by the machine precision [46, 47]. The penalty parameter $\Lambda$ should be chosen according to the rule

$$
\Lambda=c \max \{\mu, \mu R e\}
$$

where $R e$ is the Reynolds number and the constant $c$ is recommended to be about $10^{7}$ for computations with double-precision 64 bit words [47].

The Galerkin formulation of Eq. (4) and Eq. (5) can be explicitly rewritten as

$$
\begin{gathered}
{\left[\int_{\Omega} \rho N_{i}\left(\bar{u}_{i} u_{j} \frac{\partial N_{j}}{\partial x}+\bar{v}_{i} u_{j} \frac{\partial N_{j}}{\partial z}\right)\right]+\left[\int_{\Omega} \Lambda \frac{\partial N_{i}}{\partial x}\left(\frac{\partial N_{j}}{\partial x} u_{j}+\frac{\partial N_{j}}{\partial z} v_{j}\right) d \Omega\right]+} \\
{\left[\int_{\Omega} \mu\left(\frac{\partial N_{i}}{\partial x} \frac{\partial N_{j}}{\partial x}+\frac{\partial N_{i}}{\partial z} \frac{\partial N_{j}}{\partial z}\right) u_{j} d \Omega\right]=R H S_{x},} \\
{\left[\int_{\Omega} \rho N_{i}\left(\bar{u}_{i} v_{j} \frac{\partial N_{j}}{\partial x}+\bar{v}_{i} v_{j} \frac{\partial N_{j}}{\partial z}\right)\right]+\left[\int_{\Omega} \Lambda \frac{\partial N_{i}}{\partial z}\left(\frac{\partial N_{j}}{\partial x} u_{j}+\frac{\partial N_{j}}{\partial z} v_{j}\right) d \Omega\right]+} \\
{\left[\int_{\Omega} \mu\left(\frac{\partial N_{i}}{\partial x} \frac{\partial N_{j}}{\partial x}+\frac{\partial N_{i}}{\partial z} \frac{\partial N_{j}}{\partial z}\right) v_{j} d \Omega\right]=R H S_{z},}
\end{gathered}
$$

where $N_{i}$ is the weight function, $N_{j}, u_{j}, v_{j}$ are the node shape function and the velocities in each element, $\bar{u}_{i}$ and $\bar{v}_{i}$ are the lagged velocities, and the right hand side $\left(R H S_{x}, R H S_{z}\right)$ terms include the boundary velocities.

In our simulation, the bilinear quadrilateral elements $(i, j=1,2,3,4)$ with non-orthogonal edges are transformed to straight-sided orthogonal elements by introducing the natural coordinates $\xi=\xi(x, z)$ and $\eta=\eta(x, z)$. The shape functions $N_{i}$ in the natural coordinate system are defined as

$$
N_{i}=\frac{\left(1+\xi_{i} \xi\right)\left(1+\eta_{i} \eta\right)}{4} \quad i=1, . ., 4
$$

where $\xi_{i}$ and $\eta_{i}$ are the corner points of each element (see Fig.2). 
In the next step, Eq. (7) and Eq. (8) are integrated numerically using four-point Gaussian quadrature [48]. The final system of equations is constructed as follows:

$$
\left[\rho\left[K_{1}\right]+\Lambda\left[K_{2}\right]+\mu\left[K_{3}\right]\right]\left[\begin{array}{l}
u \\
v
\end{array}\right]=\left[\begin{array}{c}
R H S_{x} \\
R H S_{z}
\end{array}\right]
$$

where the terms $R H S_{x}$ and $R H S_{z}$ contain the velocities at the boundary nodes.

The boundary conditions must be specified at the inlet, outlet and upper and lower walls of the Couette cell. The periodic boundary conditions are imposed at inlet and outlet along the $\hat{x}$ direction. A finite slip is allowed along the lower wall while the boundary condition at the upper wall is always no-slip. In the local coordinate system (spanned by the tangential $\vec{t}$ and normal $\vec{n}$ vectors), the fluid velocity along the lower wavy wall is computed from

$$
u_{t}=L_{0}\left[(\vec{n} \cdot \nabla) u_{t}+u_{t} / R(x)\right]
$$

where $u_{t}$ is the tangential component of $\mathbf{u}=u_{t} \vec{t}+u_{n} \vec{n}, L_{0}$ is the intrinsic (or microscopic) slip length at the flat surface, and $R(x)$ is the local radius of curvature [26]. The radius of curvature is positive for concave and negative for convex regions. The Navier slip condition for a flat wall is recovered from Eq. (11) when $R(x) \rightarrow \infty$. The effective slip length $L_{\text {eff }}$ at the corrugated lower wall is obtained by extrapolating the linear part of the velocity profile $(0.45 \leqslant z / h \leqslant 0.9)$ to zero velocity with respect to the reference line $a=0$.

The simulation begins by setting the no-slip boundary condition at the upper and lower walls as an initial guess. Once Eq. (10) is solved, the fluid velocities at the lower boundary are updated using Eq. (11). This iteration is repeated until the solution is converged to a desired accuracy. The convergence rate of the solution remains under control by using the under-relaxation value 0.001 for the boundary nodes. The results presented in this paper are obtained with the grid resolution $150 \times 150$ in the $\hat{x}$ and $\hat{z}$ directions, respectively. In order to check the accuracy of the results, several sets of simulations were also carried out with a finer grid $180 \times 180$. The maximum relative error of the effective slip length due to the grid size is $L_{\text {eff }} / h=0.003$. The converged solution of the Navier-Stokes equation satisfies the following boundary condition:

$$
u_{t}=L_{s}(x) \frac{\partial u_{t}}{\partial n}, \quad \frac{1}{L_{s}(x)}=\frac{1}{L_{0}}-\frac{1}{R(x)}
$$

where $L_{s}(x)$ is the local slip length in the presence of surface curvature [26]. 
The accuracy of the numerical solution is checked by the normalized average error, which is defined as

$$
\text { error }=\left[\sum_{i=1}^{N_{p}} \frac{\left|u_{i}^{n}-u_{i}^{n+1}\right|}{\left|u_{i}^{n+1}\right|}\right] / N_{p},
$$

where $N_{p}$ is the total number of computational nodes, $u_{i}^{n}$ is the velocity at the node $i$ and time step $n$, and $u_{i}^{n+1}$ is the velocity in the next time step. The typical value of the error in the converged solution is less than $10^{-9}$. Throughout the study, the results are presented in the non-dimensional form. The length scale, shear rate, shear stress, and velocity are normalized by $h, \dot{\gamma}^{*}, \tau_{w}^{*}$, and $U^{*}$, respectively, where $\dot{\gamma}^{*}$ is the shear rate in the case of no-slip boundary condition at the flat upper and lower walls, and $\tau_{w}^{*}=\mu \dot{\gamma}^{*}$ and $U^{*}=h \dot{\gamma}^{*}$.

\section{RESULTS}

\section{A. Analytical solution of the Stokes equation for viscous flow over a wavy wall}

The effect of small periodic surface roughness on the effective slip length has been previously investigated for pressure-driven flows in a Couette geometry [25, 26]. The analytical solution of the Stokes equation with boundary conditions Eq. (12) at the wavy wall with amplitude $a$ and wavelength $\lambda$ was obtained for two limiting cases and small $\mathrm{ka}$. For $L_{0} / \lambda \ll 1$ the effective slip length is given by

$$
\lim _{k L_{0} \rightarrow 0} L_{e f f}=L_{0}-k a^{2} \omega_{\circ}(k a)
$$

while in the limit of $L_{0} / \lambda \gg 1$ it reduces to

$$
\lim _{k L_{0} \rightarrow \infty} L_{e f f}=\left(\frac{1}{L_{0}}+\frac{k^{3} a^{2}}{\omega_{\infty}(k a)}\right)^{-1},
$$

where the functions $\omega_{\circ}(k a)$ and $\omega_{\infty}(k a)$ are defined as

$$
\omega_{\circ}(k a)=\frac{1-1 / 4(k a)^{2}+19 / 64(k a)^{4}+O\left[(k a)^{6}\right]}{1+(k a)^{2}-1 / 2(k a)^{4}+O\left[(k a)^{6}\right]},
$$

and

$$
\omega_{\infty}(k a)=\frac{1-5 / 4(k a)^{2}+61 / 64(k a)^{4}+O\left[(k a)^{6}\right]}{1+(k a)^{2}-1 / 2(k a)^{4}+O\left[(k a)^{6}\right]} .
$$

An approximate analytical expression for the effective slip length that interpolates between the two bounds Eq. (14) and Eq. (15) is given by

$$
L_{e f f}=\frac{L_{0} \omega_{\infty}(k a)-k a^{2} \omega_{0}(k a) /\left(1+2 k L_{0}\right)}{1+k^{3} a^{2} L_{0}},
$$


with the range of applicability $k a \lesssim 0.5$. For larger wavenumbers $k a>0.5$, the function $\omega_{\infty}(k a)$ overestimates the numerical solution and the interpolated formula Eq. (18) does not apply [26].

\section{B. Flow over a rough surface with the local no-slip boundary condition}

In this section, the Stokes equation with the local no-slip condition at the upper and lower walls is solved numerically to study the effect of corrugation amplitude on the effective slip length. The velocity profiles, averaged over the period of corrugation $\lambda$, are plotted in the inset of Fig. 3 for several values of wavenumber $k a=2 \pi a / \lambda$. As $k a$ increases, the normalized velocity profiles remain linear in the bulk region and become curved near the lower corrugated wall. The linear part of the velocity profiles is used to compute the effective slip length, which is plotted as a function of wavenumber in Fig. 3. With increasing corrugation amplitude of the lower wall, the effective slip length decays monotonically and becomes negative, indicating that the effective no-slip boundary is shifted into the fluid domain. For

$k a \lesssim 1$, the numerical results agree well with the analytical solution Eq. (18) denoted by the solid line in Fig.3. The deviation from the analytical solution becomes significant at larger wavenumbers where the streamlines extracted from the Stokes solution indicate the presence of backflow at the bottom of the valley.

In order to investigate the flow behavior above the sinusoidal surface, the shear stress and pressure along the lower wall were computed from the solution of the Stokes equation. In the presence of surface curvature the wall shear stress $\tau_{w}$ has two components

$$
\tau_{w}=\left.\mu\left(\frac{\partial u_{t}}{\partial n}+u_{t} / R(x)\right)\right|_{w}
$$

where $\partial u_{t} / \partial n$ is the normal derivative of the tangential velocity $u_{t}$, and $R(x)$ is the local radius of curvature. In the case of no-slip boundary condition $\left(u_{t}=0\right)$, the local shear stress at the wall is reduced to $\tau_{w}=\mu \frac{\partial u_{t}}{\partial n}$. The normalized shear stress along the lower corrugated wall is plotted in Fig. 4 for different corrugation amplitudes. The maximum value of the shear stress is located at the peak of the surface corrugation $(x / \lambda=0.25)$ and it increases with increasing amplitude, which is consistent with the results of previous analytical studies of a laminar flow over a wavy wall [37, 49]. The fluid tangential velocity near the boundary is proportional to the wall shear stress shown in Fig.4, Therefore, the tangential velocity 
is also maximum above the peak and, as the flow moves downstream, it decelerates and the velocity becomes zero inside the valley at sufficiently large amplitudes. For $k a \geqslant 0.79$, the shear stress profiles intersect the dashed line $\left(\tau_{w}=0\right)$ at two points and a clockwise flow circulation develops inside the valley. As the corrugation amplitude increases, the intersection points move away from each other and the flow recirculation region becomes larger. These results are in agreement with previous estimates of the critical wavenumber $k a \approx 0.77$ for the onset of flow separation in sufficiently thick films [44, 45].

The pressure along the lower wavy wall is plotted in Fig.5 for the same amplitudes as in Fig.4. The value $P^{*}$ used for normalization is the maximum pressure, which is located above the wavy surface with $k a=1.12$ on the left side of the peak. For each wavenumber, the pressure along the surface is maximum on the left side of the peak, where the surface faces the mainstream flow. The surface pressure reaches its minimum value on the right side of the peak (see Fig. (5) ). As the flow moves further downstream into the valley, it encounters an adverse pressure gradient, which becomes larger as $k a$ increases. At large wavenumbers $k a \geqslant 0.83$, the flow near the surface cannot overcome the combined resistance of the viscous forces and the adverse pressure gradient, and separates from the surface at the point where $\tau_{w}=0$.

The pressure contours and streamlines near the corrugated surface with wavenumber $k a=1.12$ are depicted in Fig.6(a). The pressure contours indicate the presence of an adverse pressure gradient in the region $0.3 \lesssim x / \lambda \lesssim 0.6$ on the right side of the peak (see also Fig.(5). The streamlines illustrate the flow separation inside the valley at $x / \lambda \simeq 0.52$. After the separation point the flow near the wall reverses direction and moves against the mainstream. The local velocity profile inside the valley is shown in the inset of Fig.6(a). As the flow cross-section decreases towards the peak, the flow attaches to the surface at the point where the shear stress is zero again. Note also that for $k a \geqslant 0.83$ in Fig. 5, the pressure profiles along the lower surface exhibit a slight drop inside the valley where the vortex is present. After the attachment point, due to the periodic boundary condition in the $\hat{x}$ direction, the surface pressure increases up to its maximum value, which is located on the left side of the peak. The nonlinearity due to inertia is absent in the Stokes flow and the vortex in the valley remains symmetrical.

We also comment that a secondary vortex is formed inside a deeper cavity $(k a \approx 2.33$ ), which is counter-rotating with respect to the primary vortex (not shown). Previous an- 
alytical study of a creeping flow over a wavy wall demonstrated that a secondary vortex appears at $k a \gtrsim 2.28$ when the channel width is larger than the corrugation wavelength [45]. In the limiting case, when the cavity consists of two parallel walls, an infinite sequence of counter-rotating vortices appears between the walls [50, 51].

\section{Effect of the local slip on the flow pattern near the rough surface}

Next, we present the results of the numerical solution of the Stokes equation with the local slip condition at the lower wavy wall while the boundary condition at the upper wall remains no-slip. The pressure contours and the streamlines are plotted in Fig.6 for several values of the intrinsic slip length $L_{0}$ and the wavenumber $k a=1.12$. The streamline patterns indicate that with increasing slip length $L_{0}$, the size of vortex inside the valley is progressively reduced and the vortex eventually disappears. Similar to the analysis in the previous section, the pressure and shear stress along the lower wall are computed in the presence of the local boundary slip.

The normalized pressure along the lower wavy wall is plotted in Fig.7 as a function of the slip length $L_{0}$. Similar to the case of no-slip boundary condition, the profiles exhibit a maximum and a minimum in pressure on the right and left sides on the peak, respectively. In a wide range of $L_{0}$, the difference between the locations and the magnitudes of the extrema is barely noticeable. As the flow moves down a slope $(0.3 \lesssim x / \lambda \lesssim 0.5$ region in Fig.(7), the pressure gradient along the surface becomes positive and its magnitude decreases with increasing values of $L_{0}$. The separation and attachment points are located at the intersection of the shear stress profiles with the horizontal line $\left(\tau_{w}=0\right)$ shown in Fig.8. In comparison to the no-slip case, the smaller combined effect of the adverse pressure gradient and the wall shear stress causes a shift of the separation point deeper into the valley (e.g. see Fig.61). As the slip length $L_{0}$ increases, the separation and attachment points move closer to each other, the vortex becomes smaller and eventually vanishes. We also note that at the separation point both components of the shear stress $\partial u_{t} / \partial n$ and $u_{t} / R(x)$ become zero in agreement with the MRS criterion for the flow separation of the boundary layer at a moving substrate [52].

The influence of the local slip on the shape of the velocity profile and the normal derivative of the tangential velocity at the bottom of the valley is illustrated in the insets of Fig.6. 
The local slip length $L_{s}(x)$ in Eq. (12) is a function of the radius of curvature $R(x)$ and the intrinsic slip length $L_{0}$. For all cases considered in Fig.6, the condition $L_{0}<|R(x)|$ holds and the local slip length $L_{s}$ remains positive everywhere along the lower wall, which means that the slip velocity $u_{t}$ and the normal derivative $\partial u_{t} / \partial n$ carry the same sign. It is expected, however, that for the opposite condition, $L_{0}>|R(x)|$, the values $u_{t}$ and $\partial u_{t} / \partial n$ would have different signs at the bottom of the valley, and the corresponding velocity profile will be qualitatively similar to the profile shown in the inset of Fig.6(d) but shifted by a negative slip velocity (see next section).

The effective slip length computed from the numerical solution of the Stokes equation and Eq. (18) is plotted in Fig.9as a function of the intrinsic slip length $L_{0}$ for wavenumbers $k a=$ $0.28(a / h=0.04)$ and $k a=1.12(a / h=0.18)$. For small values of $L_{0}$, the effective slip length approaches a negative value previously reported in Fig. [3 for the no-slip case. As $L_{0}$ increases, $L_{\text {eff }}$ grows monotonically and appears to saturate to a constant value. The transition of the effective slip length from a growing function of $L_{0}$ to a nearly constant value occurs at larger $L_{0}$ when $k a$ decreases. For the small wavenumber $k a=0.28$, the effective slip length computed from Eq. (18) is in a good agreement with the numerical solution of the Stokes equation. Visual inspection of the streamline patterns indicates that there is no backflow at any $L_{0}$. In the saturation regime, $L_{0} \rightarrow \infty$, the wall shear stress becomes zero everywhere along the lower boundary, the streamlines near the lower wall follow the boundary curvature, and the effective slip length in Eq. (15) approaches $L_{\text {eff }} / \lambda \simeq 1 /\left[2 \pi(k a)^{2}\right]-9 / 8 \pi$. For the large wavenumber $k a=1.12$, the analytical results Eq. (18) overestimate $L_{\text {eff }}$ computed from the numerical solution at $L_{0} / h \gtrsim 0.02$ and the flow circulation is developed in the valley at $L_{0} / h \leqslant 0.067$. The vortex vanishes at the bottom of the valley at sufficiently large values of $L_{0}$ (denoted by the vertical arrow in Fig.9), and the flow streamlines are deformed to follow the boundary curvature [e.g. see Fig.66(d)]. The results for the intrinsic slip length, which determines the threshold for the onset of the flow circulation at the bottom of the groove, are summarized in Fig.10. For the wavenumbers examined in this study, $k a \leqslant 1.26$, the numerical simulations indicate that if the flow circulation is present in the valley then the effective slip length is negative and $L_{\text {eff }}$ increases with decreasing vortex size. 


\section{Effect of the Reynolds number on the effective slip length}

The analysis of the Stokes equation discussed in the previous section demonstrated that increasingly large local slip at the lower wavy wall eliminates the flow circulation in the valley and leads to a larger effective slip. In this section, the influence of the inertia term in the Navier-Stokes equation on the flow pattern and the effective slip length is investigated. For the shear flow with slip condition at the lower corrugated wall $L_{0}$, the Reynolds number is defined as

$$
R e=\frac{\rho U^{*} h\left(1+L_{e f f} / h\right)}{\mu},
$$

where $\rho$ is the fluid density, $U^{*}$ is the upper wall velocity, and $h\left(1+L_{\text {eff }} / h\right)$ is the distance between the upper flat wall and the effective no-slip boundary plane. In the case of no-slip boundary condition at the lower flat wall, $L_{\text {eff }}$ is zero and the standard definition of the Reynolds number is recovered, i.e., $R e=\rho U^{*} h / \mu$.

The pressure and shear stress along the lower wavy wall are plotted in Fig.11 for the selected values of the Reynolds number and no-slip boundary conditions. As Re increases, the adverse pressure gradient along the right side of the corrugation peak $(0.3 \lesssim x / \lambda \lesssim 0.5)$ becomes larger and the pressure drop inside the valley $(0.5 \lesssim x / \lambda \lesssim 0.9)$ increases. For each value of the Reynolds number in Fig.11(b), similar to the Stokes flow case, the shear stress above the peak is maximum and, as the flow moves downstream along the right side of the peak, it decelerates and eventually separates from the surface when $\tau_{w}=0$. With increasing upper wall velocity, the shear stress above the peak increases and causes the flow to decelerate faster along the right side of the peak. The separation and attachment points (determined from the condition $\tau_{w}=0$ ) move further apart from each other and the circulation region inside the valley expands [see Fig.11(b)].

The effect of the inertia term in the Navier-Stokes equation on the shape of the wall shear stress and pressure profiles can be seen in Fig.11, The shear stress profiles above the peak are not symmetric with respect to $x / \lambda=0.25$, which means that the flow decelerates faster on the right side of the corrugation peak than it accelerates on the left side. Also, the average adverse pressure gradient on the left side of the peak is larger than its value on the right side of the peak $(0.3 \lesssim x / \lambda \lesssim 0.5)$. By increasing the upper wall velocity, the separation point moves further upstream than the attachment point downstream. The formation of asymmetric vortex flow at finite Reynolds numbers is consistent with previous findings for a 
flow in an undulated tube [36, 39, 40, 42]. The pressure contours and streamlines extracted from the NS equation with the no-slip boundary condition are plotted in Fig.12(a) for $k a=1.12$ and $R e=79$. The flow streamlines in the valley indicate an asymmetric clockwise circulation, which is larger than the flow circulation region shown in Fig.6(a) for the Stokes case.

In the presence of the local slip condition along the lower corrugated wall, the size of the vortex becomes smaller while the flow structure remains asymmetric (see Fig.12). The decrease of the vortex size is similar to the case of the Stokes flow shown in Fig. 6] and can also be described in terms of the pressure and shear stress along the lower wall. In Fig.13 the pressure and shear stress profiles are plotted for the same values of the upper wall velocity as in Fig.11 but with the slip boundary condition $\left(L_{0} / h=0.25\right)$ along the lower wall. Note that the Reynolds numbers in Fig.13 are slightly larger than the values reported in Fig.11 for the same $U^{*}$ because of the larger effective slip length entering the definition of the Reynolds number [see Eq. (20)]. For each value of the upper wall velocity, the adverse pressure gradient and the wall shear stress on the right side of the peak are smaller than in the case of the no-slip boundary condition, and, as a result, the vortex either becomes smaller or vanishes (see Figs.11 and 13). As the Reynolds number increases, however, the vortex forms and expands asymmetrically to fill the bottom of the groove. The inset of the Fig.12(b) demonstrates that the slip velocity at the bottom of the valley is negative while its normal derivative $\partial u_{t} / \partial n$ is positive, in contrast to the velocity profiles shown in Fig.6 for the same $k a=1.12$ and smaller slip lengths $L_{0} / h \leqslant 0.08$.

The effective slip length is plotted in Fig.14 as a function of the Reynolds number for the selected values of the intrinsic slip length $L_{0}$ and $k a=1.12$. With increasing Reynolds number, the flow streamlines move away from the lower boundary and straighten out, the slope of the normalized velocity profiles increases and the effective no-slip boundary plane is shifted into the fluid domain. For $L_{0} / h \leqslant 0.067$, the circulation is always present in the valley and the flow streamlines in the bulk of the fluid do not penetrate deep into the valley [e.g. see Fig.12(b)]. For larger slip lengths $L_{0} / h>0.067$, the flow streamlines show that there is no backflow at low $R e$, and the vortex is formed at the bottom of the valley only at sufficiently large Reynolds numbers indicated by the dashed line in Fig. 14. The numerical results obtained from the solution of the Navier-Stokes equation demonstrate that the growth or decay of the vortex as a function of the Reynolds number or the intrinsic slip length is 
accompanied by the decrease or increase of the effective slip length.

\section{CONCLUSIONS}

In this paper the effects of local slip boundary condition and the Reynolds number on the flow structure near sinusoidally corrugated surfaces and the effective slip length were investigated numerically by solving the Stokes and Navier-Stokes equations. The effective slip length was defined with respect to the mean height of the surface roughness by extrapolating the linear part of the velocity profile averaged over the corrugation period. In the case of the Stokes flow with the local no-slip boundary condition, the effective slip length decreases with increasing corrugation amplitude and the vortex flow develops in the groove of the rough surface for $k a \geqslant 0.79$. In the presence of the local slip boundary condition along the wavy wall, the effective slip length increases and the size of the recirculation zone is reduced. The vortex vanishes at sufficiently large values of the intrinsic slip length. The analysis of the pressure and wall shear stress computed from the Navier-Stokes equation shows that the asymmetric vortex flow develops in the groove due to the inertia term even when the local slip boundary condition is applied. The effective slip length decreases with increasing Reynolds number. The numerical simulations suggest that the variation of the vortex size as a function of either the Reynolds number or the intrinsic slip length correlates with the magnitude of the effective slip length.

\section{Acknowledgments}

Financial support from the Petroleum Research Fund of the American Chemical Society is gratefully acknowledged. Computational work in support of this research was performed at Michigan State University's High Performance Computing Facility.

[1] D. J. Beebe, G. A. Mensing, and G. M. Walker, "Physics and applications of microfluidics in biology," Annu. Rev. Biomed. Eng. 4, 261 (2002).

[2] T. M. Squires and S. R. Quake, "Microfluidics: Fluid physics at the nanoliter scale," Rev. Mod. Phys. 77, 977 (2005). 
[3] V. Mengeaud, J. Josserand, and H. H. Girault, "Mixing processes in a zigzag microchannel: Finite element simulations and optical study," Anal. Chem. 74, 4279 (2002).

[4] A. Gigras and S. Pushpavanam, "Early induction of secondary vortices for micromixing enhancement," Microfluid. Nanofluid. 5, 89 (2008).

[5] H. M. Metwally and R. M. Manglik, "Enhanced heat transfer due to curvature-induced lateral vortices in laminar flows in sinusoidal corrugated-plate channels," Int. J. Heat Mass Transfer 47, 2283 (2004).

[6] P. E. Geyer, N. R. Rosaguti, D. F. Fletcher, and B. S. Haynes, "Thermohydraulics of squaresection microchannels following a serpentine path," Microfluid. Nanofluid. 2, 195 (2006).

[7] N. R. Rosaguti, D. F. Fletcher, and B. S. Haynes, "Low-Reynolds number heat transfer enhancement in sinusoidal channels," Chem. Eng. Sci. 62, 694 (2007).

[8] J. H. Forrester and D. F. Young, "Flow through a converging-diverging tube and its implications in occlusive vascular disease. 1. Theoretical development," J. Biomech. 3, 297 (1970).

[9] D. M. Wootton and D. N. Ku, "Fluid mechanics of vascular systems, diseases, and thrombosis," Annu. Rev. Biomed. Eng. 1, 299 (1999).

[10] S. A. Berger and L. D. Jou, "Flows in stenotic vessels," Annu. Rev. Fluid Mech. 32, 347 (2000).

[11] N. V. Churaev, V. D. Sobolev, and A. N. Somov, "Slippage of liquids over lyophobic solidsurfaces," J. Colloid. Interface Sci. 97, 574 (1984).

[12] R. G. Horn, O. I. Vinogradova, M. E. Mackay, and N. Phan-Thien, "Hydrodynamic slippage inferred from thin film drainage measurements in a solution of nonadsorbing polymer," J. Chem. Phys. 112, 6424 (2000).

[13] R. Pit, H. Hervet, and L. Leger, "Direct experimental evidence of slip in hexadecane: Solid interfaces," Phys. Rev. Lett. 85, 980 (2000).

[14] C. H. Choi, K. J. A. Westin, and K. S. Breuer, "Apparent slip flows in hydrophilic and hydrophobic microchannels," Phys. Fluids 15, 2897 (2003).

[15] C. Cottin-Bizonne, B. Cross, A. Steinberger, and E. Charlaix, "Boundary slip on smooth hydrophobic surfaces: Intrinsic effects and possible artifacts," Phys. Rev. Lett. 94, 056102 (2005).

[16] O. I. Vinogradova and G. E. Yakubov, "Surface roughness and hydrodynamic boundary conditions," Phys. Rev. E 73, 045302 (2006). 
[17] P. A. Thompson and M. O. Robbins, "Shear flow near solids: Epitaxial order and flow boundary conditions," Phys. Rev. A 41, 6830 (1990).

[18] P. A. Thompson and S. M. Troian, "A general boundary condition for liquid flow at solid surfaces," Nature (London) 389, 360 (1997).

[19] J.-L. Barrat and L. Bocquet, "Large slip effect at a nonwetting fluid-solid interface," Phys. Rev. Lett. 82, 4671 (1999).

[20] V. P. Sokhan, D. Nicholson, and N. Quirke, "Fluid flow in nanopores: An examination of hydrodynamic boundary conditions," J. Chem. Phys. 115, 3878 (2001).

[21] N. V. Priezjev and S. M. Troian, "Molecular origin and dynamic behavior of slip in sheared polymer films," Phys. Rev. Lett. 92, 018302 (2004).

[22] N. V. Priezjev, "Rate-dependent slip boundary conditions for simple fluids," Phys. Rev. E 75, 051605 (2007).

[23] N. V. Priezjev and S. M. Troian, "Influence of periodic wall roughness on the slip behaviour at liquid/solid interfaces: molecular-scale simulations versus continuum predictions," J. Fluid Mech. 554, 25 (2006).

[24] A. Niavarani and N. V. Priezjev, "Rheological study of polymer flow past rough surfaces with slip boundary conditions," J. Chem. Phys. 129, 144902 (2008).

[25] D. Einzel, P. Panzer, and M. Liu, "Boundary condition for fluid flow: curved or rough surfaces," Phys. Rev. Lett. 64, 2269 (1990).

[26] P. Panzer, M. Liu, and D. Einzel, "The effects of boundary curvature on hydrodynamic fluid flow: calculation of slip lengths," Int. J. Mod. Phys. B 6, 3251 (1992).

[27] S. Richardson, "On the no-slip boundary condition," J. Fluid Mech. 59, 707 (1973).

[28] L. M. Hocking, "A moving fluid interface on a rough surface," J. Fluid Mech. 76, 801 (1976).

[29] K. M. Jansons, "Determination of the macroscopic (partial) slip boundary condition for a viscous flow over a randomly rough surface with a perfect slip microscopic boundary condition," Phys. Fluids 31, 15 (1988).

[30] M. J. Miksis and S. H. Davis, "Slip over rough and coated surfaces," J. Fluid Mech. 273, 125 (1994).

[31] E. O. Tuck and A. Kouzoubov, "A laminar roughness boundary condition," J. Fluid Mech. 300, 59 (1995).

[32] K. Sarkar and A. Prosperetti, "Effective boundary conditions for Stokes flow over a rough 
surface," J. Fluid Mech. 316, 223 (1996).

[33] C. Y. Wang, "Flow over a surface with parallel grooves," Phys. Fluids 15, 1114 (2003).

[34] N. Lecoq, R. Anthore, B. Cichocki, P. Szymczak, and F. Feuillebois, "Drag force on a sphere moving towards a corrugated wall," J. Fluid Mech. 513, 247 (2004).

[35] C. Kunert and J. Harting, "Roughness induced boundary slip in microchannel flows," Phys. Rev. Lett. 99, 176001 (2007).

[36] J. C. F. Chow and K. Soda, "Laminar flow in tubes with constriction," Phys. Fluids 15, 1700 (1972).

[37] G. L. Bordner, "Nonlinear analysis of laminar boundary layer flow over a periodic wavy surface," Phys. Fluids 21, 1471 (1978).

[38] S. Taneda, "Visualization of separating Stokes flows," J. Phys. Soc. Jpn. 46, 1935 (1979).

[39] I. Sobey, "On flow through furrowed channels. Part 1. Calculated flow patterns," J. Fluid Mech. 96, 1 (1980).

[40] T. Nishimura, Y. Ohori, and Y. Kawamura, "Flow characteristics in a channel with symmetric wavy wall for steady flow," J. Chem. Eng. Japan 17, 466 (1984).

[41] S. Tsangaris and D. Potamitis, "On laminar small Reynolds-number flow over wavy walls," Acta Mechanica 61, 109 (1986).

[42] G. Leneweit and D. Auerbach, "Detachment phenomena in low Reynolds number flows through sinusoidally constricted tubes," J. Fluid Mech. 387, 129 (1999).

[43] H. Zhou, R. J. Martinuzzi, R. E. Khayat, A. G. Straatman, and E. Abu-Ramadan, "Influence of wall shape on vortex formation in modulated channel flow," Phys. Fluids 15, 3114 (2003).

[44] C. Pozrikidis, "Creeping flow in two-dimensional channels," J. Fluid Mech. 180, 495 (1987).

[45] M. Scholle, A. Wierschem, and N. Aksel, "Creeping films with vortices over strongly undulated bottoms," Acta Mechanica 168, 167 (2004).

[46] J. C. Heinrich and R. S. Marshall, "Viscous incompressible flow by a penalty function finite element method," Computers and Fluids 9, 73 (1981).

[47] T. J. R. Hughes, W. K. Liu, and A. Brooks, "Finite element analysis of incompressible viscous flows by the penalty function formulation," J. Comp. Phys. 30, 1 (1979).

[48] J. C. Heinrich and D. W. Pepper, Intermediate finite element method: Fluid flow and heat transfer applications (Taylor and Francis, Philadelphia, 1999).

[49] T. B. Benjamin, "Shearing flow over a wavy boundary," J. Fluid Mech. 6, 161 (1959). 
[50] F. Pan and A. Acrivos, "Steady flows in rectangular cavities," J. Fluid Mech. 28, 643 (1967).

[51] H. K. Moffatt, "Viscous and resistive eddies near a sharp corner," J. Fluid Mech. 18, 1 (1964).

[52] H. Schlichting and K. Gersten, Boundary-Layer Theory (Springer, Berlin, 2000). 
Figures 


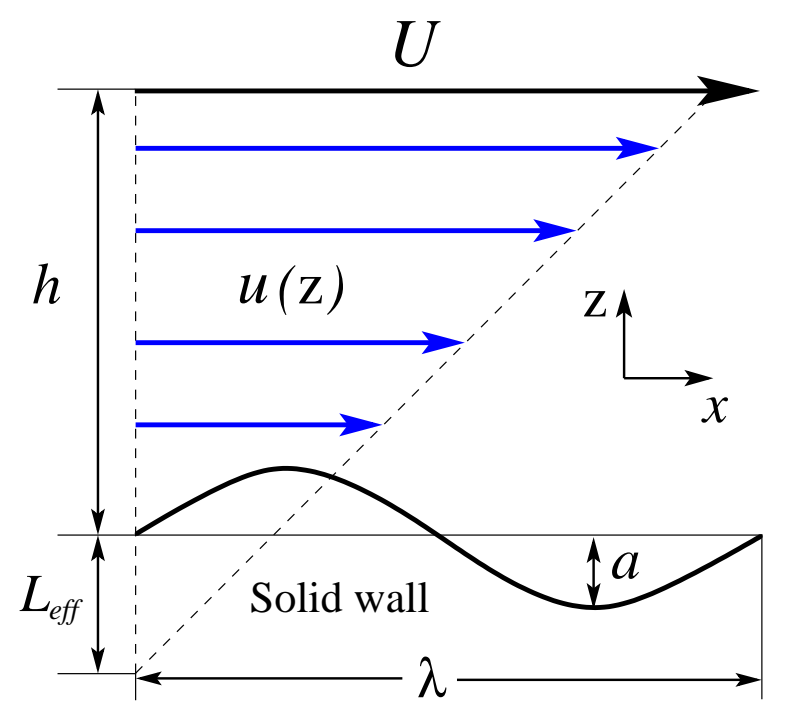

FIG. 1: (Color online) Schematic diagram of the steady-state Couette flow over a rough surface. The upper flat wall is moving with a constant velocity $U$ in the $\hat{x}$ direction. The lower stationary wall is modeled as a sinusoidal wave with amplitude $a$ and wavelength $\lambda$. The wavenumber $k a=2 \pi a / \lambda$ varies in the range $0 \leqslant k a \leqslant 1.26$. 
(a)

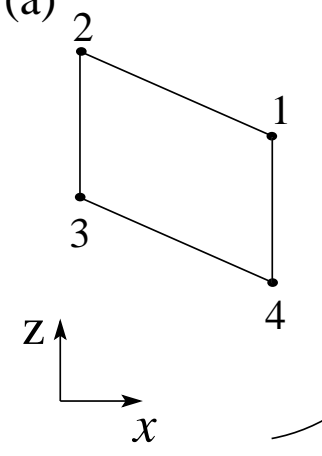

(b)

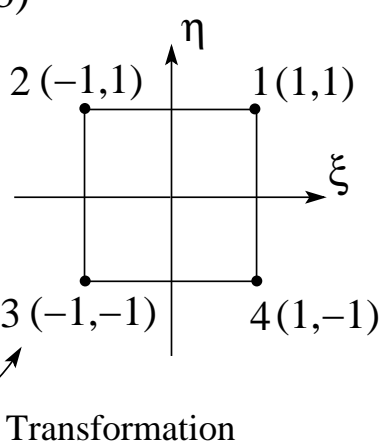

FIG. 2: Diagram of a bilinear element in (a) the physical coordinate system $(x, z)$ and (b) a transformed element in the natural coordinate system $(\xi, \eta)$. 


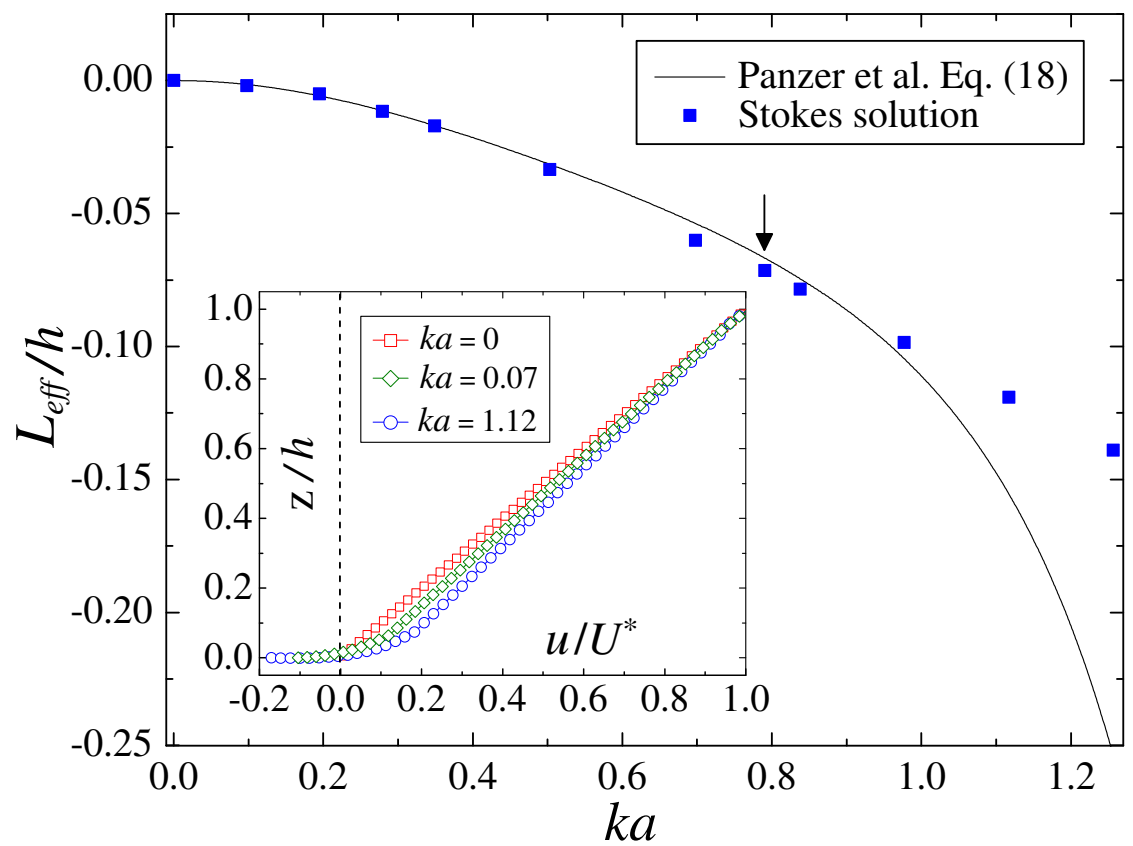

FIG. 3: (Color online) The effective slip length as a function of wavenumber $k a$ computed from the solution of the Stokes equation with the no-slip boundary condition. The primary vortex is formed at the bottom of the valley for $k a \geqslant 0.79$ (see the vertical arrow at $k a=0.79$ ). The solid line is calculated using Eq. (18). The inset shows the normalized velocity profiles obtained from the Stokes solution for the selected values of $k a$. The dashed line located at $a=0$ is the reference for calculating the effective slip length. 


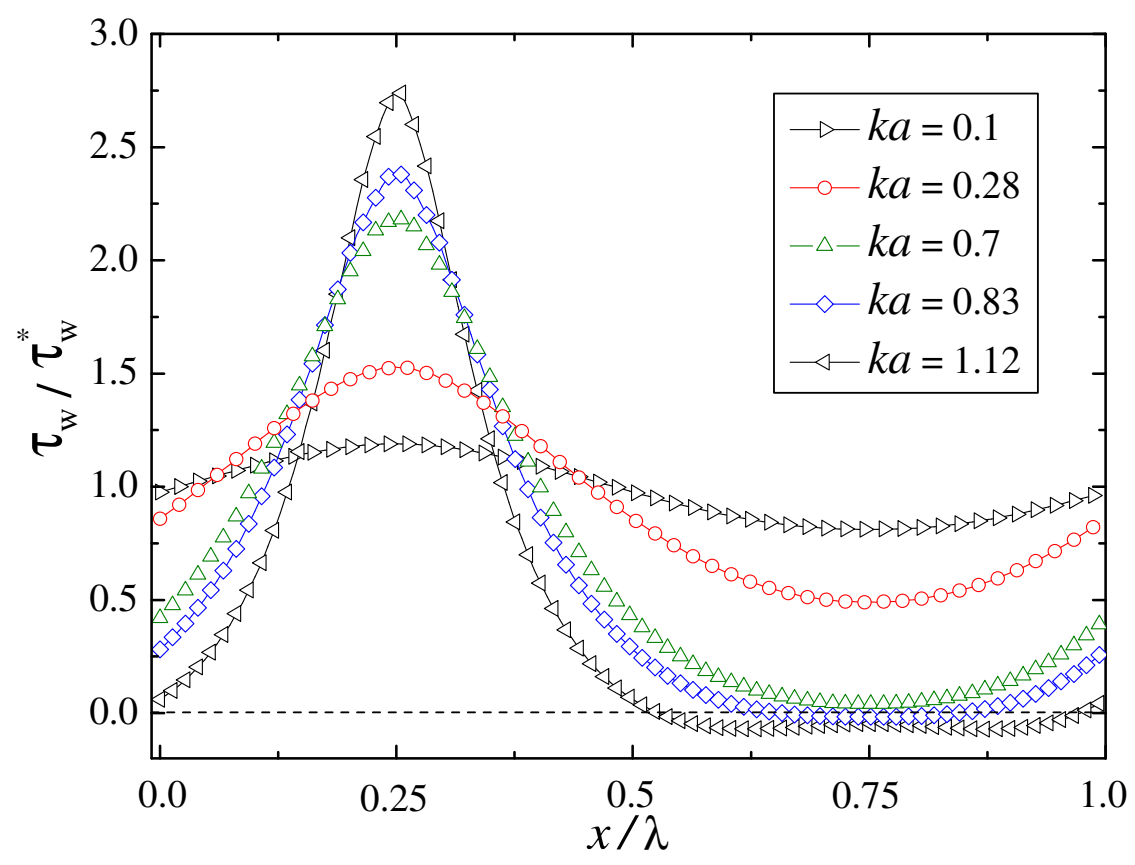

FIG. 4: (Color online) Shear stress along the lower wavy wall computed from the Stokes solution with no-slip boundary condition $\left(L_{0}=0\right)$ for the indicated values of wavenumber $k a$. The value $\tau_{w}^{*}$ used for normalization is the shear stress at the flat wall. The intersection of the curves with the dashed line determines the location of the flow separation and attachment inside the valley. 


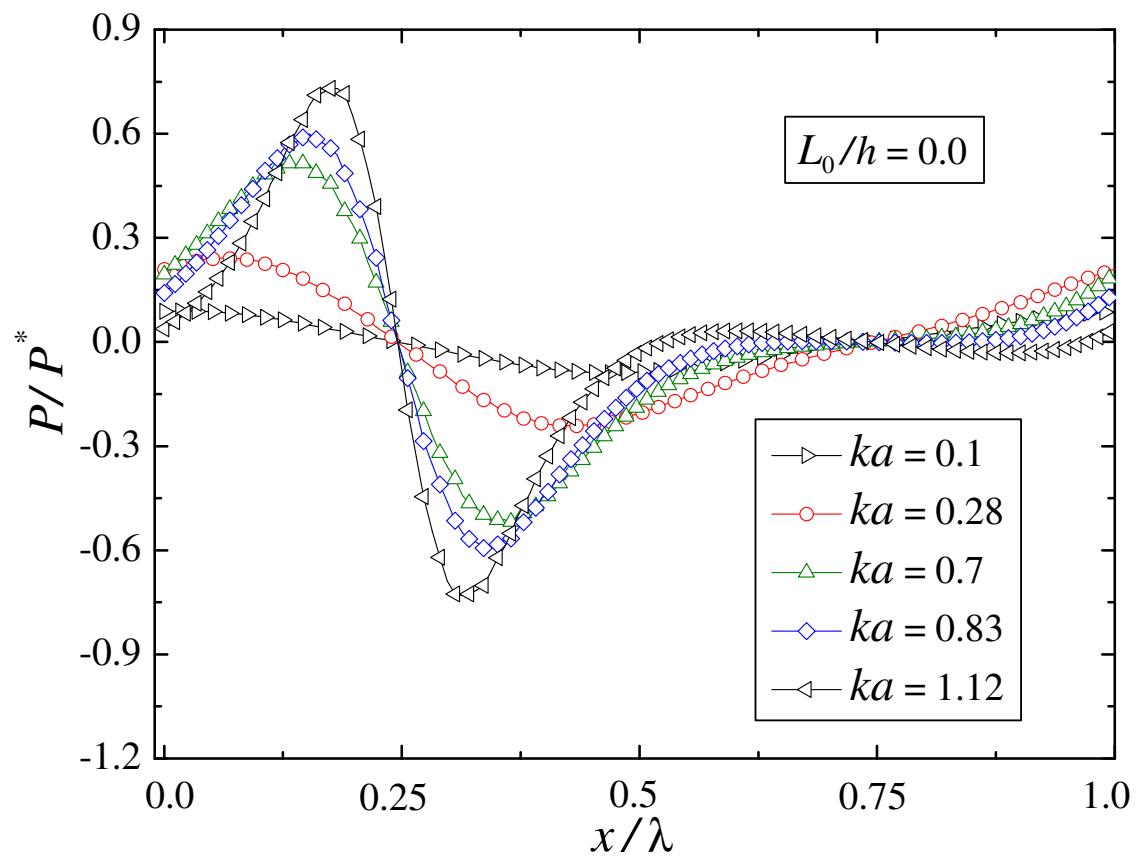

FIG. 5: (Color online) The normalized pressure along the lower corrugated boundary extracted from the Stokes solution with no-slip boundary condition for the same values of $k a$ as in Fig.4. The maximum pressure $P^{*}$ for $k a=1.12$ and $L_{0}=0$ is located in the bulk region at $(x / \lambda, z / \lambda) \simeq$ $(0.07,0.23)$. 

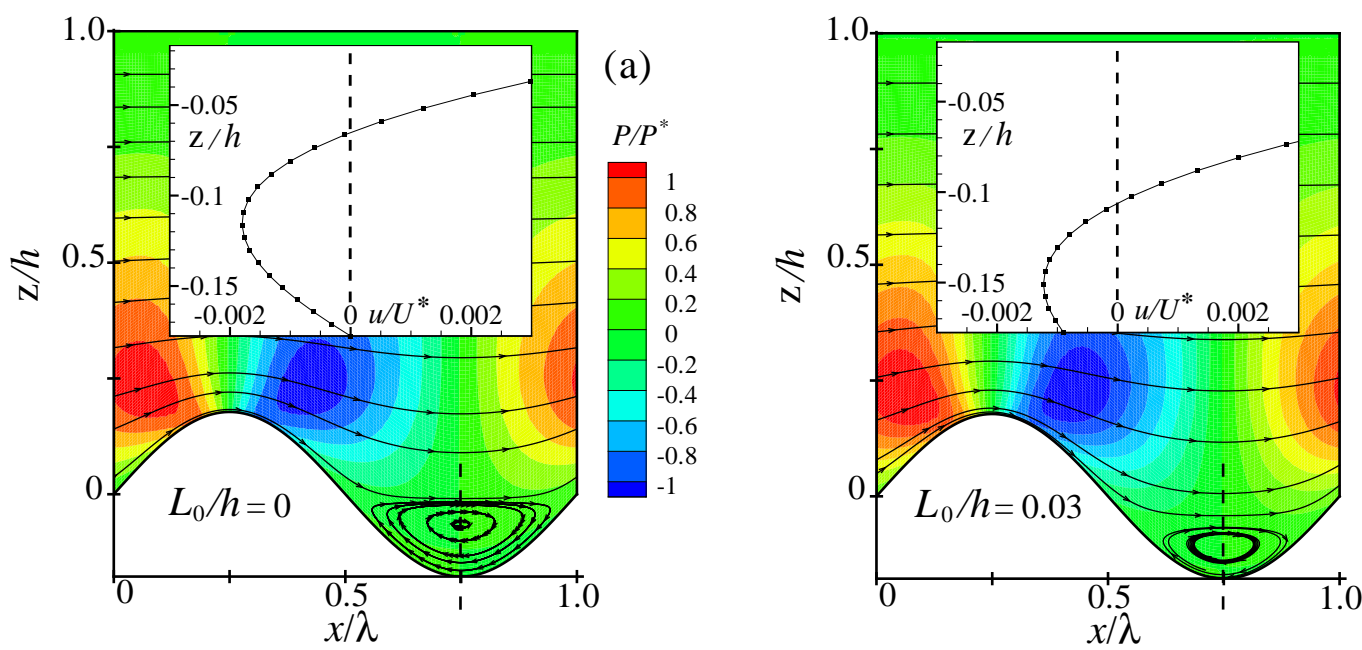

(b)
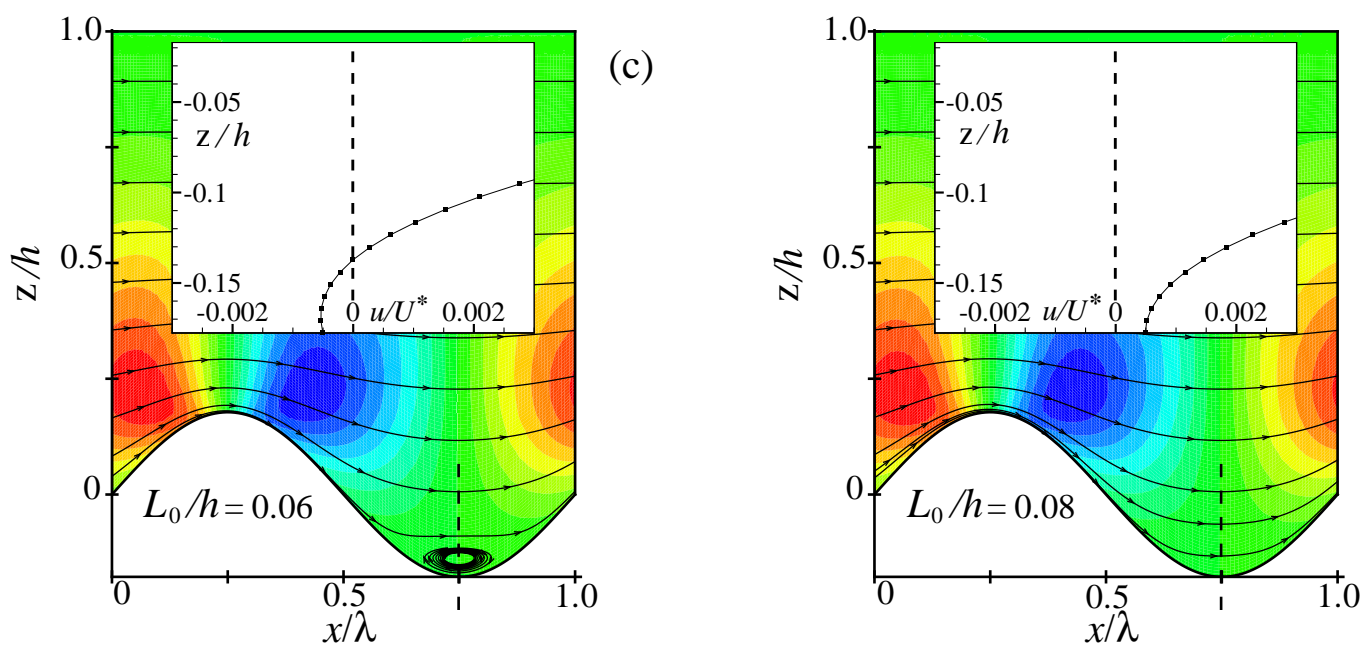

(d)

FIG. 6: (Color online) Pressure contours and streamlines for the wavenumber $k a=1.12$ and the slip length at the lower wall $L_{0}=0$ (a), $L_{0} / h=0.03$ (b), $L_{0} / h=0.06$ (c), and $L_{0} / h=0.08$ (d). The pressure contours are normalized by the maximum value $P^{*}$ located on the left side of the peak $(x / \lambda, z / \lambda) \simeq(0.07,0.23)$ in the case of $L_{0}=0$. The vertical dashed line inside the valley at $x / \lambda=0.75$ indicates the cross-section used to compute the velocity profiles shown in the inset. 


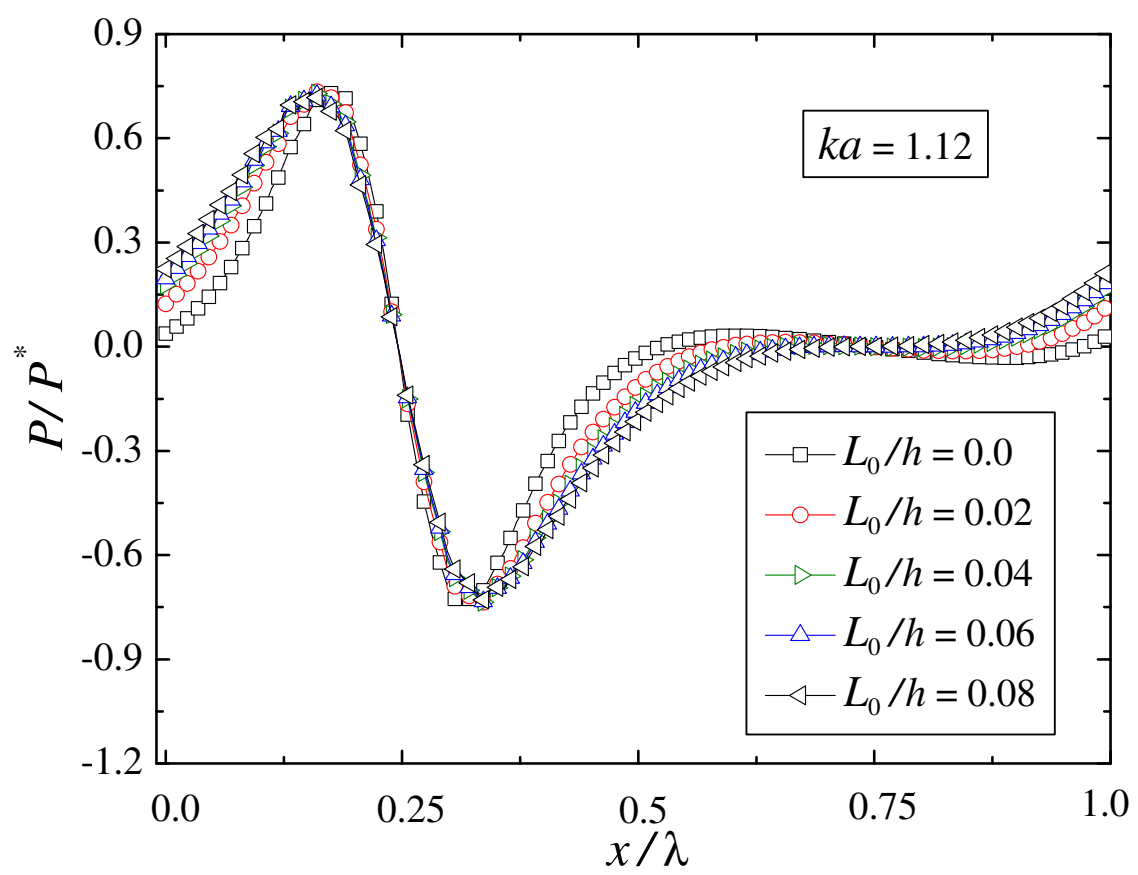

FIG. 7: (Color online) Normalized pressure along the lower wavy wall extracted from the solution of the Stokes equation for the tabulated values of $L_{0}$ and $k a=1.12$. The maximum pressure $P^{*}$ for $L_{0}=0$ is located above the lower surface at $(x / \lambda, z / \lambda) \simeq(0.07,0.23)$. 


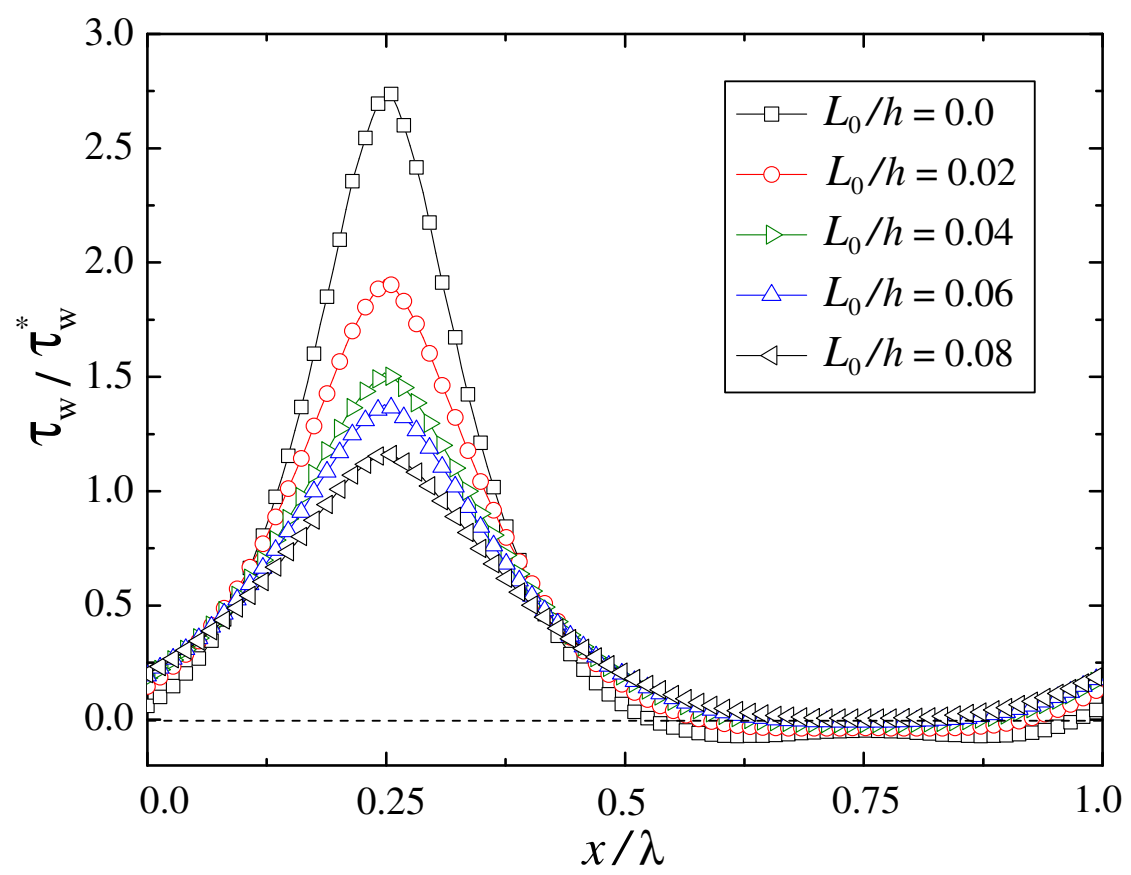

FIG. 8: (Color online) Shear stress along the corrugated lower wall $(k a=1.12)$ for the indicated values of the local slip length $L_{0}$. The intersection of the dashed line with the shear stress profiles shows the location of the flow separation and attachment inside the valley. 


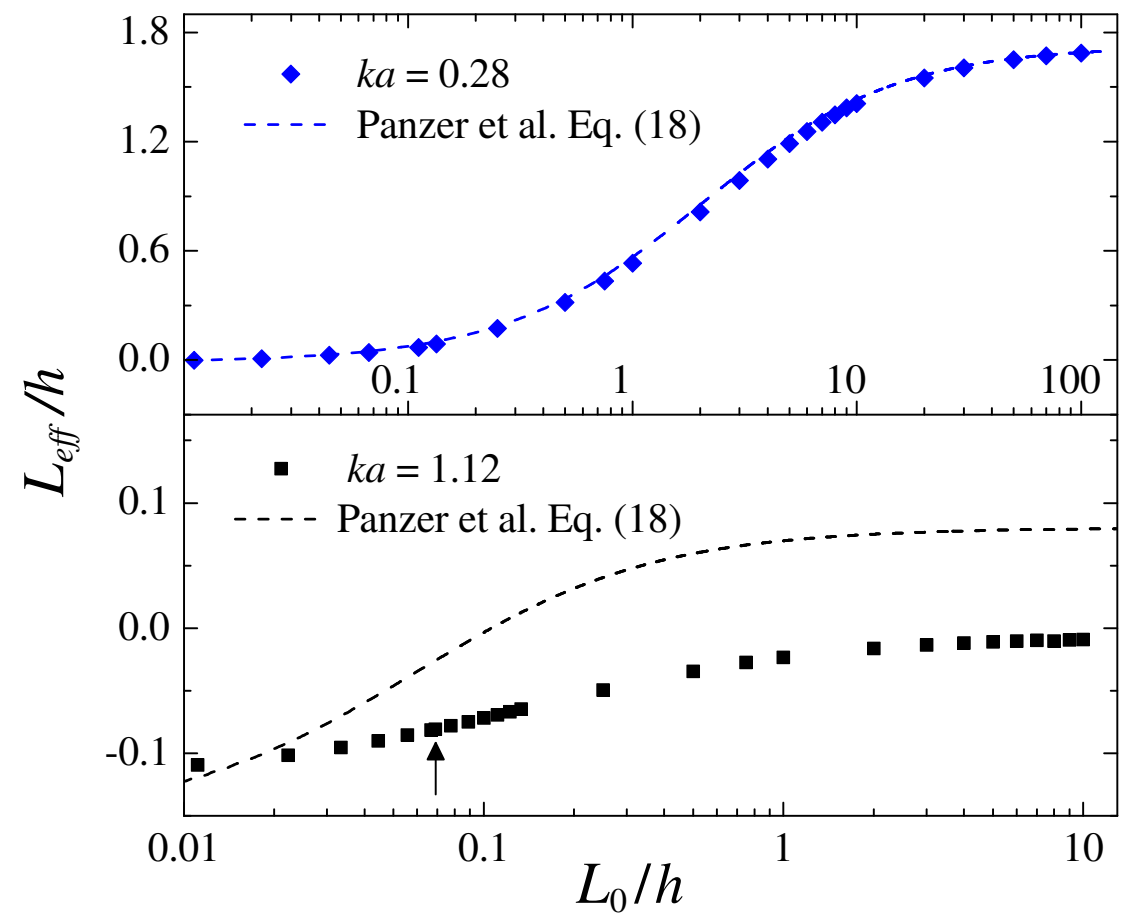

FIG. 9: The effective slip length, extracted from the Stokes solution as a function of $L_{0}$ for $k a=0.28$ (top) and $k a=1.12$ (bottom). The dashed line is computed from Eq. (18). For $k a=1.12$ and $L_{0} / h \leqslant 0.067$, the vortex is formed in the valley (see the vertical arrow at $L_{0} / h=0.067$ ). The error bars are smaller than the symbol size. 


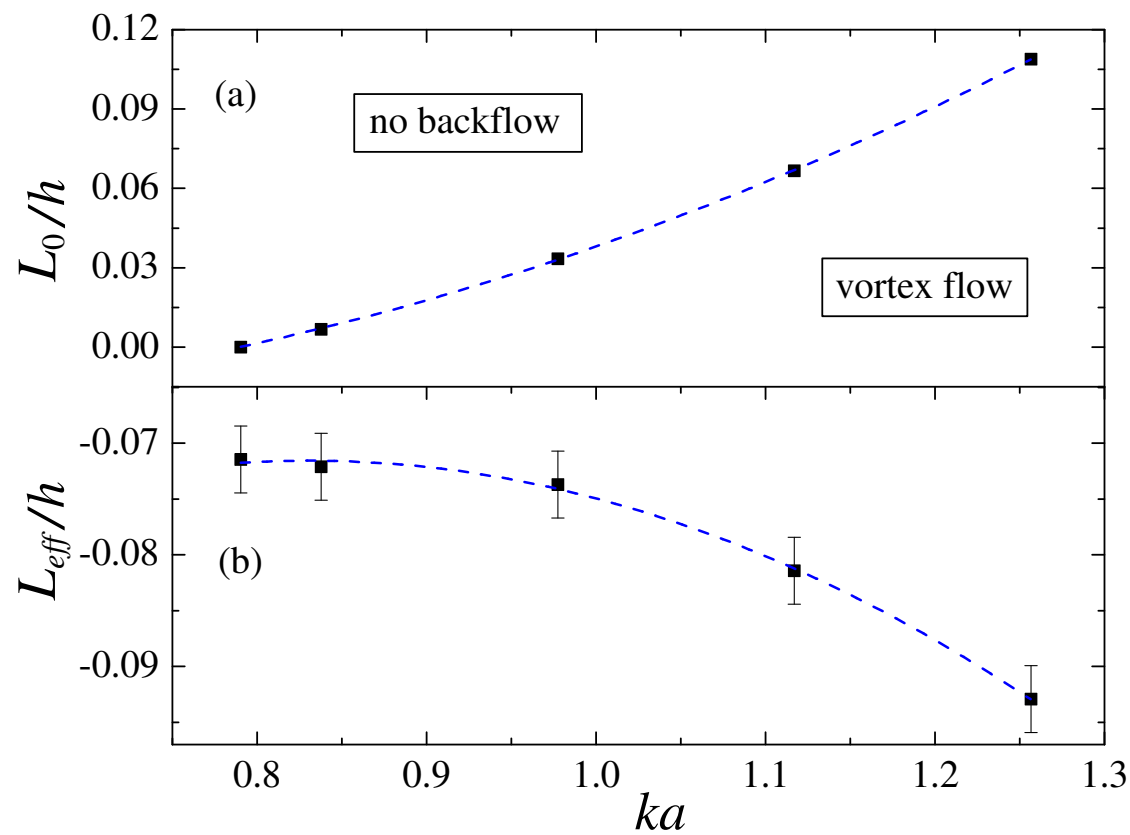

FIG. 10: (Color online) The intrinsic slip length $L_{0}$ above which there is no vortex at the bottom of the valley (top) and the corresponding effective slip length $L_{\text {eff }}$ (bottom) computed from the Stokes solution and plotted as a function of wavenumber $k a$. The increment in the slip length used to determine the threshold values is about the symbol size (top). The dashed lines are quadratic fits to guide the eye. 


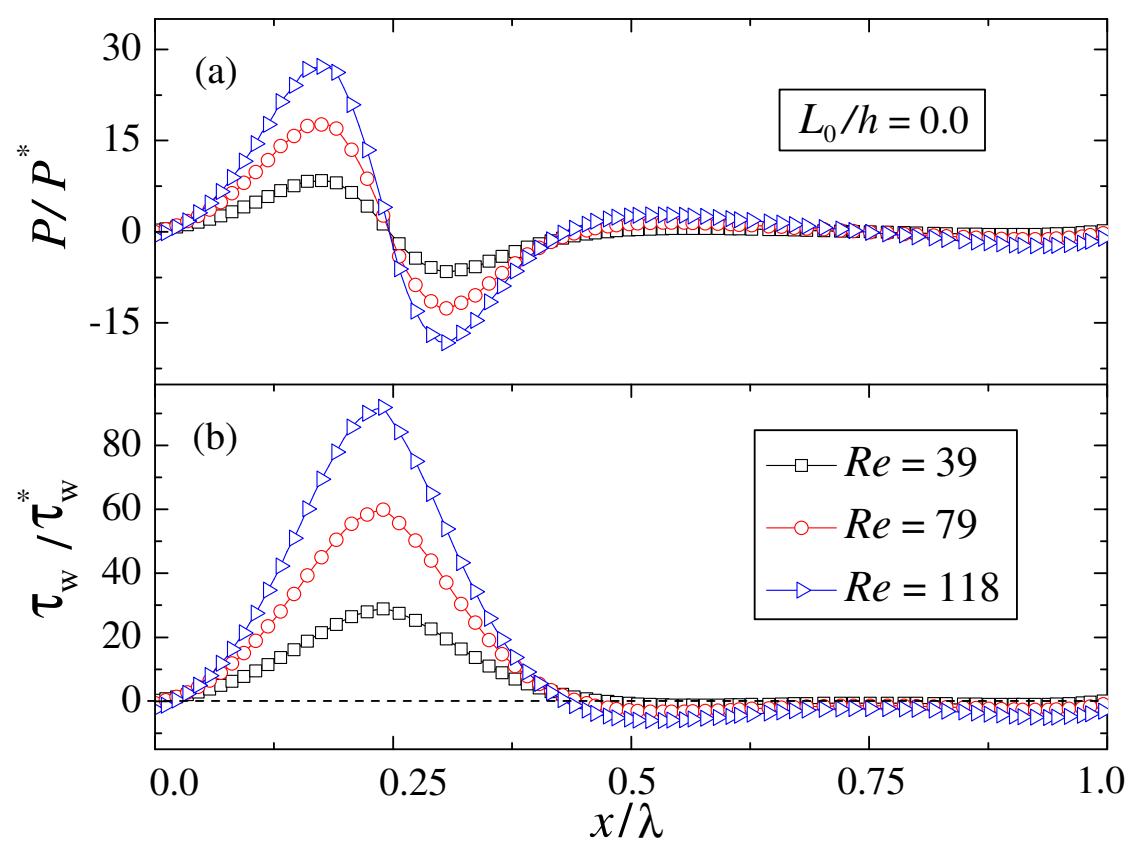

FIG. 11: (Color online) The normalized pressure (top) and shear stress (bottom) along the lower wavy wall as a function of the Reynolds number for $k a=1.12$ and no-slip boundary conditions. The value $P^{*}$ is the maximum pressure located above the lower boundary on the left side of the peak $(x / \lambda, z / \lambda) \simeq(0.07,0.23)$ for $L_{0}=0$ and $R e=4.0$. 

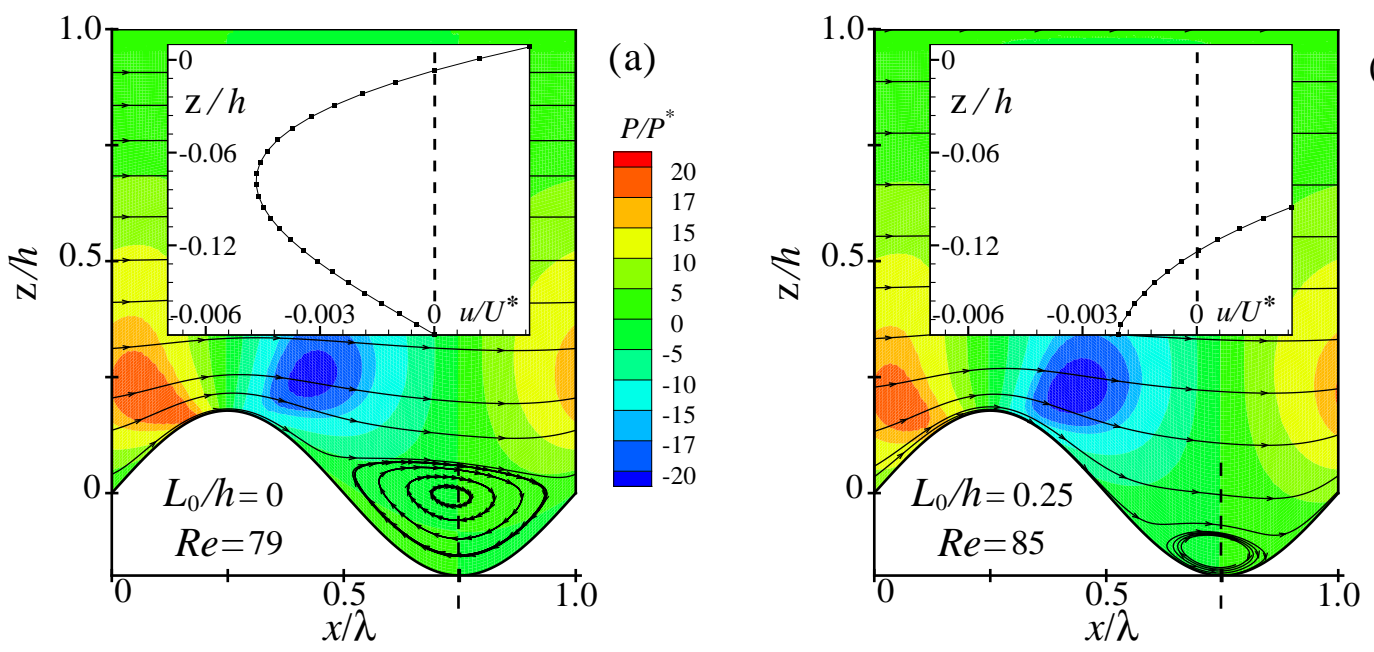

(b)

FIG. 12: (Color online) Pressure contours and streamlines extracted from the solution of the Navier-Stokes equation for $k a=1.12$ and $L_{0}=0$ (a) and $L_{0} / h=0.25$ (b). The value $P^{*}$ is the maximum pressure located at $(x / \lambda, z / \lambda) \simeq(0.07,0.23)$ for $k a=1.12, L_{0}=0$, and $R e=4.0$. The dashed line inside the valley indicates the cross-section used to compute the velocity profiles shown in the inset. 


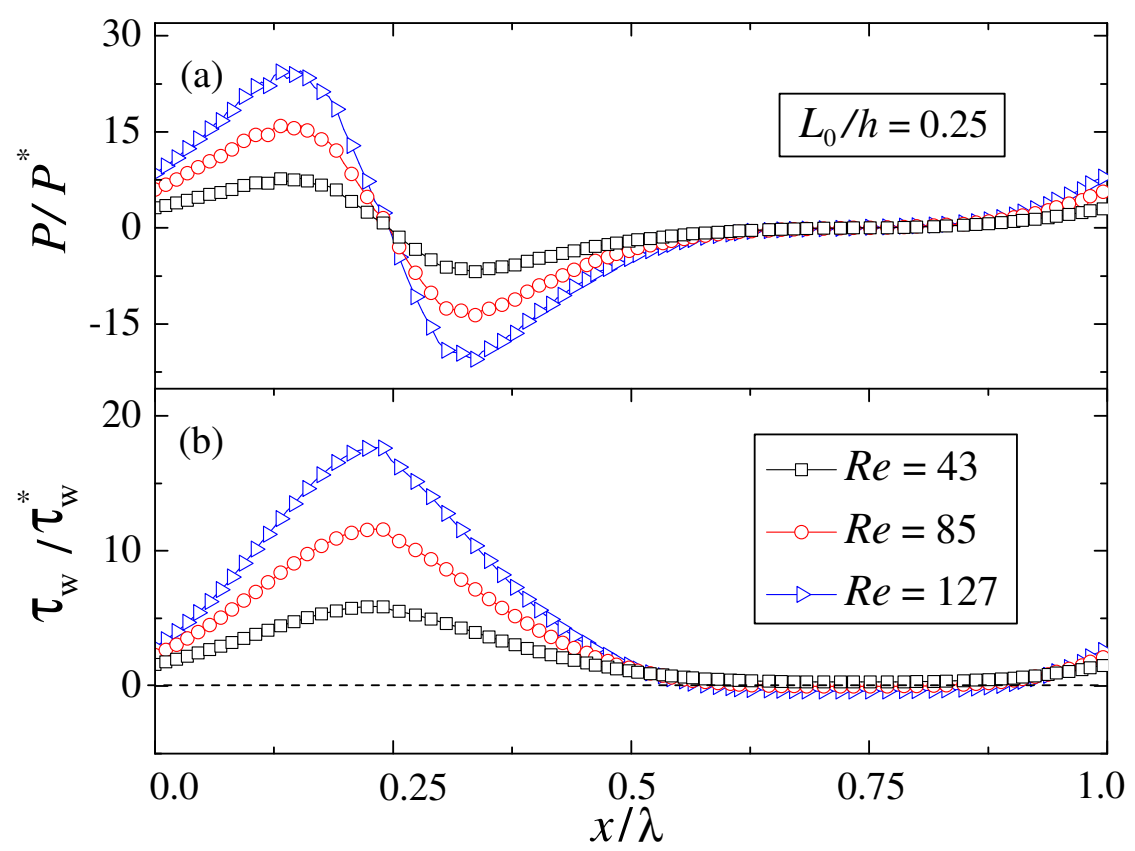

FIG. 13: (Color online) The normalized pressure (top) and shear stress (bottom) along the lower wavy wall as a function of the Reynolds number for $k a=1.12$ and $L_{0} / h=0.25$. The normalization value $P^{*}$ is the maximum pressure located above the surface on the left side of the peak $(x / \lambda, z / \lambda) \simeq$ $(0.07,0.23)$ for $L_{0}=0$ and $R e=4.0$. 


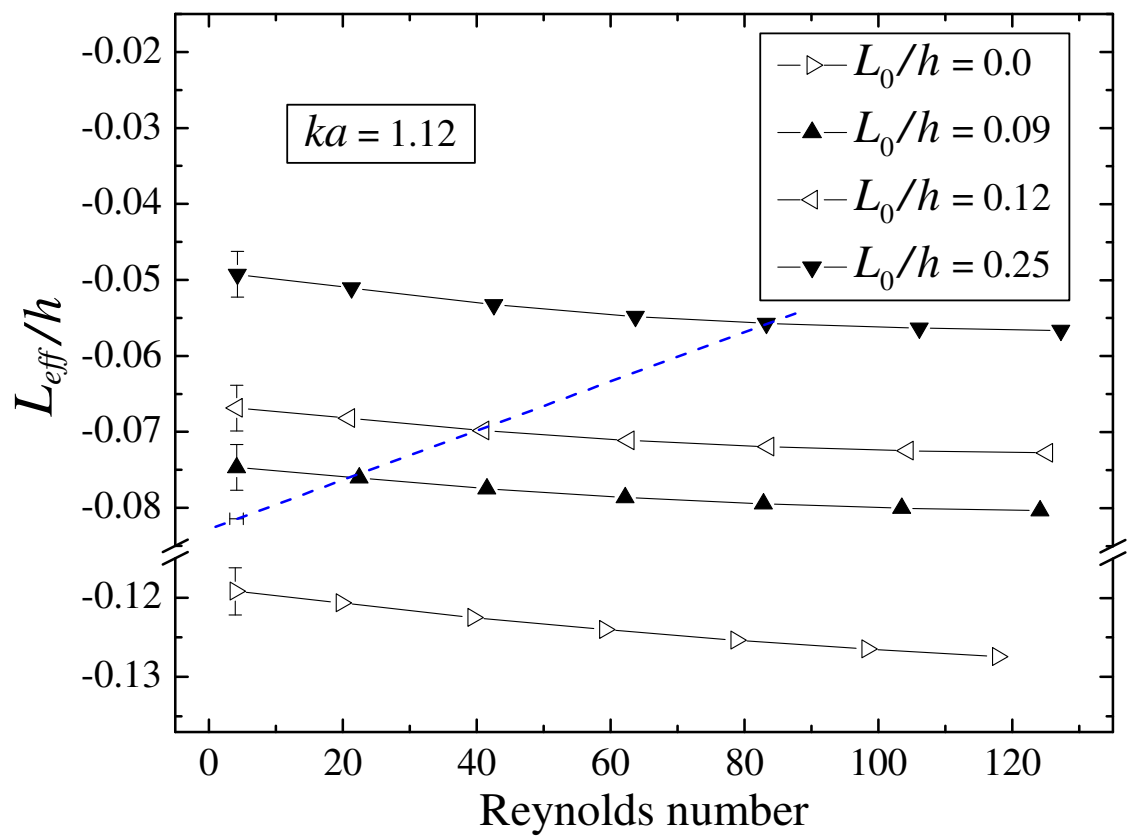

FIG. 14: (Color online) The effective slip length computed from the solution of the Navier-Stokes equation as a function of the Reynolds number for $k a=1.12$ and $L_{0}=0(\triangleright), L_{0} / h=0.09(\mathbf{\Delta})$, $L_{0} / h=0.12(\triangleleft)$, and $L_{0} / h=0.25(\mathbf{v})$. The dashed line indicates the upper bound of the region where a vortex is present in the groove of a rough surface. The error bars associated with the threshold values of the Reynolds number are about the symbol size. 